\title{
Denoising of Additive Noise in DECT scan and MRI Image using ANW filter
}

\author{
Ashwini N M $\mathbf{M}^{1}$, Kavitha $\mathbf{G}^{\mathbf{2}}$ \\ M.Tech Student, DOS in Computer Science and Engineering, UBDTCE, Davangere ${ }^{1}$ \\ Assistant Professor, DOS in Computer Science and Engineering, UBDTCE, Davangere 2
}

\begin{abstract}
Noise reduction in material density images is a necessary pre-processing step for the correct interpretation of dual-energy computed tomography (DECT) images and MRI scanned images. In this paper a new method based on a local adaptive processing to reduce noise in DECT and MRI images will be described. Here the comparative study is made based on the filters used for denoising the image. The methods used to describe the systems are Adaptive Neighbourhood Wiener(ANW) filter, prewitt and anisotropic are used among this which one is best method can be determined based on the Peak Signal Noise Ratio (PSNR) value and Mean Squarer Error (MSE) value of the input image for each filters. In this we added Gaussian noise to DECT scanned images and MRI scanned images for the noisy image we apply this filters to denoise the images. Finally by taking the PSNR average value of the each filter we can conclude which filters is best.
\end{abstract}

Keywords: Noise reduction, Preprocessing, DECT, MRI, prewitt, anisotropic, PSNR, ANW filter.

\section{INTRODUCTION}

Noise reduction is the process of removing noise from signal. Noise reduction is one of the pre-processing steps involved in image processing. Noise represents unwanted information which deteriorates image quality. Noise is random variation of image intensity and visible as the image. Noise means, pixel within the picture present different intensity value rather than correct pixel value. Noise originates from the physical nature of detection processes and has many specific forms and causes. Computed tomography (CT) scanning is used in medical field to identify the infected part in the body. With continued advances in computed tomography (CT), Dual Energy CT (DECT) as emerged as a promising technologic development with numerous applications pertinent to abdominal imaging.

The techniques used in this paper are ANWF, Prewitt and Anisotropic all this methods are used to remove noise from the DECT scanned and MRI scanned images. We can determine the noise removal from the image by calculating the PSNR value of the image. Here we added Gaussian noise to the scanned images and we calculate the PSNR and MSE value of the noisy image. After that we apply different filters to that noisy images then we get denoised image which reduces the noise from the scanned image.

Adaptive noise correction of dual energy computed tomography images which was proposed by the author Rafael S M, christlan J. In this paper the author used ANW filter for DECT images, here we have applied the ANW filter for DECT scanned and MRI scanned images. And we have applied Prewitt and Anisotropic filters for both DECT and MRI scanned images.

\section{PROBLEM STATEMENT}

In the last three decades, many different technical solutions for the acquisition of DECT images have been attempted two of these technologies are currently mature and being sold commercially. The single-source rapid voltage switching approaches by general electric (ge) and the dual-source approach by siemens. Due to slightly different spectra, these two approaches have some intrinsic and important differences in the resulting images. While dect does not have spectrographic qualities, the two basis material decomposition processes are flexible enough and capable of providing density information of many different pairs of basis materials. Unfortunately, that process results in images with significant more noise than in regular ct images. Since noisier images may negatively affect diagnoses, a preprocessing step that minimizes the noise is necessary.

\section{PROPOSED SYSTEM}

In this project the noise correction of material density images and virtual monochromatic images obtained from DECT data sets explored. Our technique takes into consideration both single- and dual-source scanners. It employs an ANW filter and anisotropic filter to eliminate the noise in the material density images. The system performance is measured 
by calculating the PSNR and MSE value of the scanned images. In this we added Gaussian noise to image and we apply filters to remove Gaussian noise.

\section{RELATED WORK}

Dual-energy computed tomography is affected by the same noise artifacts that are found in regular CT. Therefore, most of the noise problems that affect the acquisition of a single linear attenuation image can be resolved with the same mature methods (for example, scanner calibration) that are applied to regular CT. Furthermore, it is known that noiserelated errors that cause uncertainties in the measured attenuation values can be enhanced or decreased by an appropriate choice of image reconstruction algorithms and convolution kernels. Recently, the introduction of iterative and statistical reconstruction algorithms has provided radiologists with high-quality images, while, at the same time, decreasing the total dose a patient is exposed to. However, they may increase the reconstruction time. More advanced reconstruction algorithms are still being actively investigated. In this paper, we limit this session to noise correction as a post reconstruction steps.

\section{METHODOLOGY}

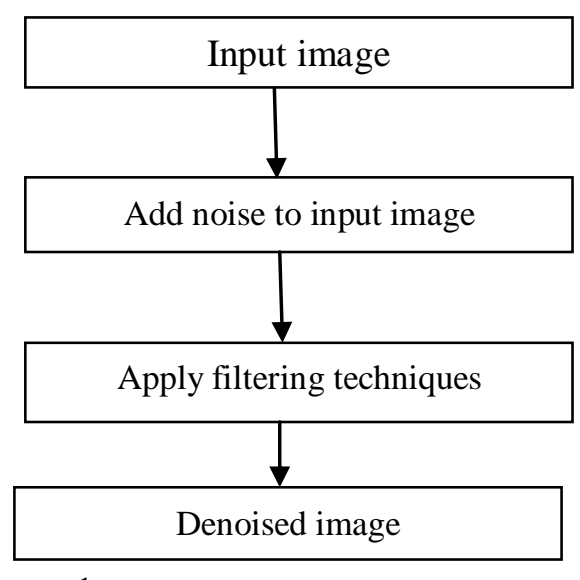

FIG 1: FLOWCHART OF THE SYSTEM

In this paper the system is developed using matlab tool, the experiment is conducted by taking set of images. In this paper the input images are taken and Gaussian noise is added to the image after that PSNR value and MSE value of the noisy image is calculated. Next for that noisy image apply all the filtering techniques to remove noise from the image. Before calculating the PSNR value the system will calculate the MSE value using the formulas.

$$
\text { MSE }=\operatorname{sum}\left(\operatorname{sum}\left((\text { InputImage-ReconstructedImage })^{\wedge} 2\right)\right) /\left(M^{*} \mathrm{~N}\right)
$$

Where, InputImage is the original image, ReconstractedImage is the noise added image, $\mathrm{M}$ and $\mathrm{N}$ represents the number of rows and columns. The PSNE value is calculated using the formula.

$$
\mathrm{PSNR}=10 * \log 10(256 * 256 / \mathrm{MSE})
$$

Contrary to the work of Balda et al. [1] and Borsdorf et al.[2], where linear attenuation or HUimages are corrected first, our focus lies primarily on the noise correction of material density, followed by the correction of virtual monochromatic images in HU. The most common image size for CT images is $512 \times 512$ pixels. We apply our ANW filter followed by an anisotropic diffusion filter at each wavelet level and again at the original image size. At each wavelet level, after the application of the Wiener filter and the anisotropic diffusion filter, we calculate an intermediate noise map by subtracting the filtered material density image from the original image, creating a correlated noise map and adding back a weighted version of that map to each material density image. In order to measure the level of image quality improvement achieved by the new algorithm and for comparison reasons, we also modify the algorithm, employing the classic Wiener filter in the form of the Lee filter instead of the newly proposed adaptive Wiener filter.

The Gaussian noise

Gaussian noise is statistical noise having a probability density function (PDF) equal to that of the normal distribution, which is also known as the Gaussian distribution. The values that the noie can take on are Gaussian distributed. The probability density function $\mathrm{p}$ of a Gaussian random variable $\mathrm{z}$ is given by:

$$
P G(z)=\frac{1}{\sigma \sqrt{2} \Pi} e^{\wedge} \frac{(z-\mu)^{\wedge} 2}{2 \sigma^{\wedge} 2}
$$


Where $\mathrm{z}$ represents the grey level, the mean value and $\sigma$ the standard deviation.

The adaptive Wiener filter

We modified the Lee filter so it employs an adaptive region growing method in its calculation of the mean value of a region. Instead of calculating the local statistics of noise and signal within a fixed window size and fixed window shaped local neighborhood, we employ an adaptive region growing technique that, given some constrains, finds an near-optimal, variable size and variable shape neighborhood for each individual pixel in the image. This method was inspired by the work of Rangayyan et al. , who used a similar idea for natural images. We adapt and extend their idea to DECT material density images and take advantage of the edges obtained from the linear attenuation image with minimal noise to restrict growth in certain areas.

$$
W(f 1, f 2)=\frac{H *(f 1, f 2) \operatorname{Sxx}(f 1, f 2)}{|H(f 1, f 2)|^{2} \operatorname{Sxx}(f 1, f 2)+\operatorname{Snn}(f 1, f 2)}
$$

where $\operatorname{Sxx}(\mathrm{f} 1, \mathrm{f} 2)$, Snn(f1,f2)are respectively power spectra of the original image and the additive noise, and $\mathrm{H}(\mathrm{f} 1, \mathrm{f} 2)$ is the blurring filter.

The Prewitt edge detection

The prewitt operator is used in image processing, particularly within edge detection algorithm. Technically, it is a discrete differentiation operator, computing an approximation of the gradient of the image intensity function. The operator uses two $3 * 3$ kernels which are convolved with the original image to calculate approximations of the derivatives- one for horizontal changes and one for vertical. If we define A as the source image, and Gx and Gy are two images which at each point contain the horizontal and vertical derivative approximations,

$$
\mathrm{G}=\sqrt{\mathrm{Gx}^{2}+\mathrm{Gy}^{2}}
$$

Using this information, we can also calculate the gradient direction:

$$
\theta=\operatorname{atan} 2(\mathrm{Gx}, \mathrm{Gy})
$$

Where, for example, $\Theta$ is 0 for a vertical edge which is darker on the right side.

The Anisotropic filter

Anisotropic filter is a method of enhancing the image quality of textures on surface of computer graphics that are at oblique's viewing angle with respect to the camera where the projection of the texture appears to be non-orthogonal. In image processing and computer vision, anisotropic diffusion, also called person malik diffusion, is a technique aiming at reducing image noise without removing significant parts of the image content, typically edges, lines or other details that are important for the interpretation of the images.

$$
\frac{\partial \mathrm{I}}{\partial \mathrm{t}}=\operatorname{div}(\mathrm{c}(\mathrm{x}, \mathrm{y}, \mathrm{t}) \nabla \mathrm{I})=\nabla \mathrm{c} . \nabla \mathrm{I}+\mathrm{c}(\mathrm{x}, \mathrm{y}, \mathrm{t}) \Delta \mathrm{I}
$$

Where, $\Delta$ denotes the laplacian, $\nabla$ denotes the gradient, $\operatorname{div}(\ldots)$ is the divergence operator and $\mathrm{c}(\mathrm{x}, \mathrm{y}, \mathrm{t})$ is the diffusion coefficient. $\mathrm{c}(\mathrm{x}, \mathrm{y}, \mathrm{t})$ controls the rate of diffusion .

\section{VI.RESULTS}

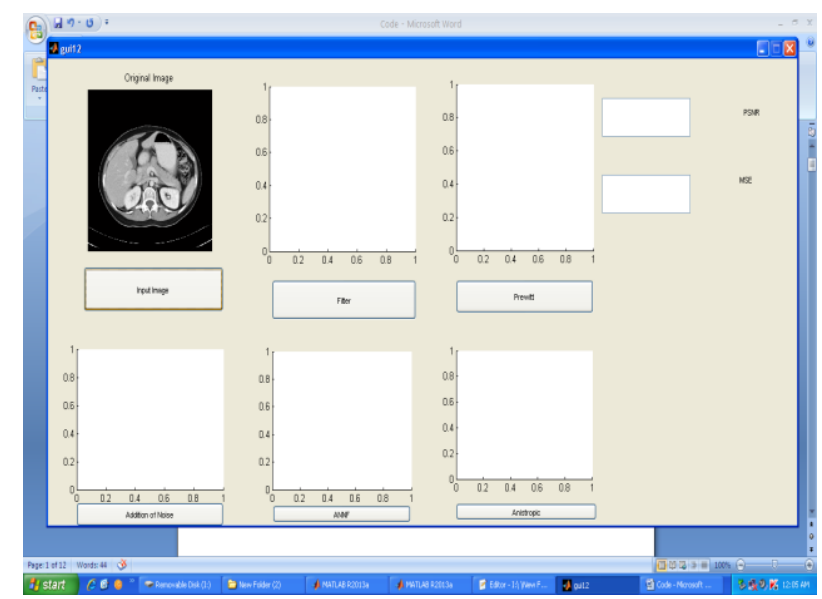

Fig (a) 
Vol. 6, Issue 6, June 2017

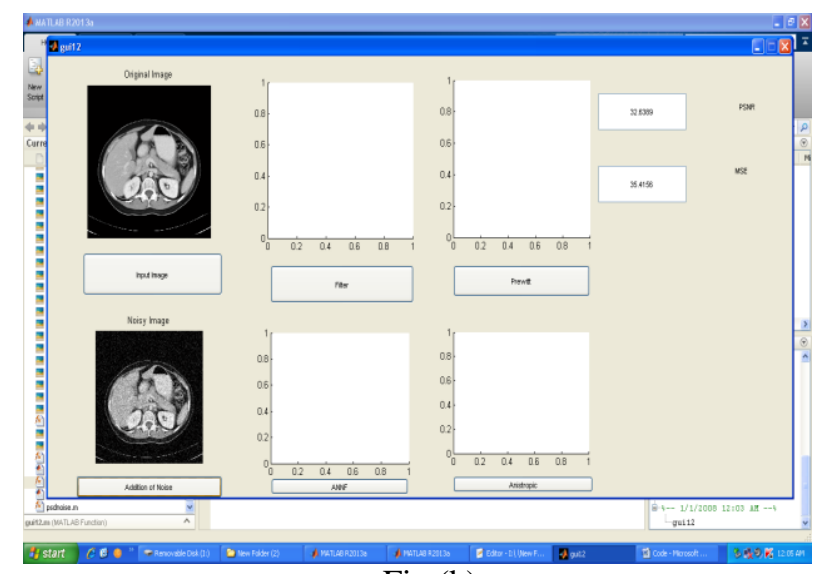

Fig (b)

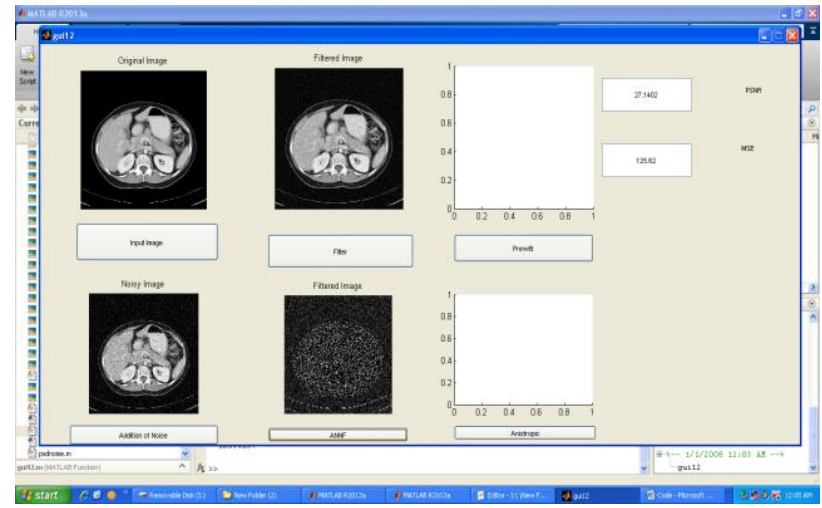

Fig (c)

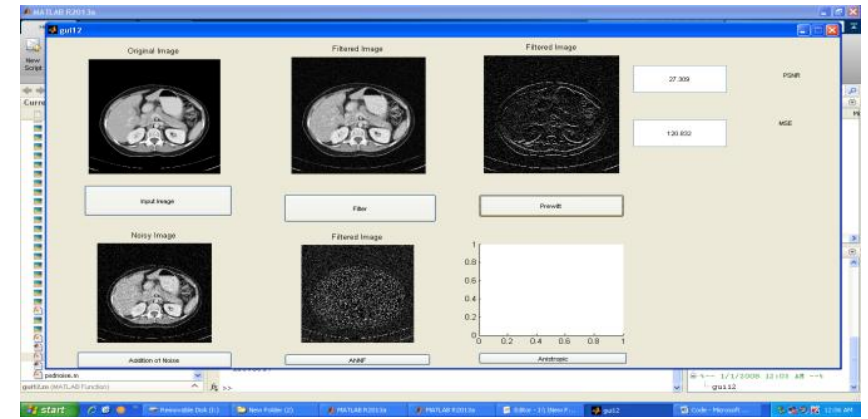

Fig (d)

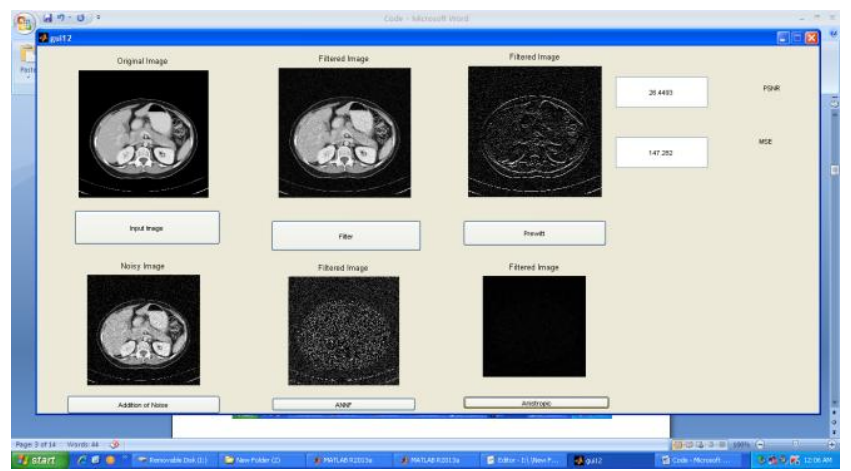

Fig (e)

Here we can observe the results of this system, fig (a) shows the input image given to the system, fig (b) shows the Gaussian noise added to the input image, fig (c) shows the ANW filter applied to the noisy image and its PSNR value is displayed at the left side to the window, fig (d) displays the prewitt edge detection and fig (e) shows the anisotropic filter. 
Vol. 6, Issue 6, June 2017

Table 1 : Table shows the PSNR values DECT images

\begin{tabular}{|c|c|c|c|}
\hline Sl.no & ANWF & Prewitt & Anisotropic \\
\hline 1 & 70.5772 & 48.34268 & 52.74992 \\
\hline 2 & 85.1384 & 48.73542 & 37.20872 \\
\hline 3 & 62.6460 & 48.7529 & 48.0091 \\
\hline 4 & 91.7081 & 48.3922 & 34.7373 \\
\hline
\end{tabular}

Table 2 : Table shows the PSNR values MRI scanned images

\begin{tabular}{|c|c|c|c|}
\hline Sl.no & ANWF & Prewitt & Anisotropic \\
\hline 1 & 61.5332 & 48.5267 & 39.1047 \\
\hline 2 & 72.0326 & 48.6008 & 37.9737 \\
\hline 3 & 70.8861 & 48.7096 & 36.5262 \\
\hline 4 & 58.2683 & 48.544 & 36.0409 \\
\hline
\end{tabular}

\section{CONCLUSION}

The quality of the material density images and virtual mono-chromatic images. Our algorithm's key innovations lie in (1) the usage of a customized adaptive Wiener filter, developed specifically for DECT material density images, and (2) adaptations required keeping details and avoiding over-blurring of the image when combined with the anisotropic filter. In this paper the Gaussian noise is added to DECT and MRI scanned images and PSNR value is calculated to noisy images after calculating PSNR value different filters are applied to noisy images to get the denoised images. Finally we can observe which method is best for noise reduction is by calculating the PSNR value of the images. From the result we can see that ANW filter has higher value, so compare to all the filters.

\section{REFERENCES}

1. Balda M, Heismann B, Hornegger J (2010) Value-based noise reduction for low-dose dual-energy computed tomography. Springer, Berlin

2. BorsdorfA,Raupach R, Flohr T, Hornegger J (2008)Wavelet based noise reduction in CT-images using correlation analysis. IEEE Trans Med Imaging 27(12):1685-1703

3. Grant KL, Flohr TG, Krauss B, Sedlmair M (2014) Assessment of an advanced image-based technique to calculate virtual monoenergetic computed tomographic images from a dual-energy examination to improve contrast-to-noise ratio in examinations using iodinated contrast media. Investig Radiol 49(9):586-592

4. Hinshaw DA, Dobbins JT III (1995) Recent progress in noise reduction and scatter correction in dual-energy imaging. In: Van Metter RL, Beutel J (eds) Medical imaging. SPIE, Washington, pp 134-142

5. Kalender W, Klotz E, Kostaridou L (1988) An algorithm for noise suppression in dual energy CT material density images. IEEE Trans Med Imaging 7(3):218-224

6. Lee JS (1980)Digital image enhancement and noise filtering by use of local statistics. IEEE Trans Pattern Anal Mach Intell 2:165-168

7. Macovski A, Nishimura DG, Doost-Hoseini A, Brody WR (1983) Measurement-dependent filtering: a novel approach to improved SNR. IEEE Trans Med imaging 2(3):122-127

8. Maia RS, Jacob C, Hara AK, Silva AC, Pavlicek W, Ross MJ(2015) An algorithm for noise correction of dual-energy computed tomography material density images. Int J Comput Assist Radiol Surg 10(1):87-100

9. Rafael Simon Maia, Christian Jacob, Amy K. Hara, Alvin C Silva, William Pavlicek, J. Ross Mitchell (2016) Adaptive noise correction of dual energy computed tomography images. Int J CARS (2016) 11:667-678 